\title{
Abnormal N-Glycosylation of Human Lens Epithelial Cells in Type-2 Diabetes May Contribute to Cataract Progression
}

This article was published in the following Dove Press journal: Clinical Ophthalmology

\author{
Ivan Ramos-Martínez ${ }^{1-3}$ \\ Oscar Vivanco-Rojas ${ }^{2}$ \\ Brenda Juárez-Domínguez ${ }^{2}$ \\ Luis Hernández-Zimbrón ${ }^{1,2}$ \\ Lenin Ochoa-de la Paz ${ }^{1,2}$ \\ Hugo Quiroz-Mercado ${ }^{2}$ \\ Eleazar Ramírez-Hernández (D) \\ Rosario Gulias-Cañizo (iD ${ }^{4}$ \\ Edgar Zenteno ${ }^{1,2}$
}

'Departamento de Bioquímica, Facultad de Medicina UNAM, Ciudad de Mexico, 04510, Mexico; ${ }^{2}$ Departamento de Investigación, Asociación para Evitar la Ceguera en Mexico I.A.P. Hospital Dr. Luis Sánchez Bulnes, Mexico City, Mexico; ${ }^{3}$ Glycobiology, Cell Growth and Tissue Repair Research Unit (GlyCRRET), Université Paris Est Créteil (UPEC), Créteil, France; ${ }^{4}$ Centro de Investigación en Ciencias de la Salud (CICSA), Universidad Anáhuac Mexico, Huixquilucan, Estado de Mexico, Mexico

Correspondence: Rosario Gulias-Cañizo; Edgar Zenteno

Email rosariogulias@yahoo.com.mx; ezenteno@servidor.unam.mx
Purpose: In order to better understand cataract development, we analyzed the glycosylation profile of human lens epithelial cells (HLECs) from anterior lens capsules of type 2 diabetes mellitus (T2DM) and non-diabetic (ND) patients undergoing routine cataract surgery.

Setting: Research Department of the Asociación para Evitar la Ceguera, Hospital "Dr. Luis Sánchez Bulnes”, Mexico.

Design: Experimental study.

Methods: Evaluation of anterior lens capsules from T2DM and ND patients undergoing phacoemulsification and free from other ocular diseases.

Results: Hematoxylin-eosin staining revealed HLECs alterations in T2DM samples. From lectins with different sugar specificities used, concanavalin A showed significant differences, labeling homogeneously both in the cytoplasm and in cell membranes in ND capsules, while in T2DM capsules, in addition to membrane and cytoplasm labeling, there were perinuclear vesicles with high concanavalin A labeling. Two-dimensional gel electrophoresis showed that T2DM patients have a $\sim 65-\mathrm{kDa}$ spot with an isoelectric point of 5.5 with a higher density compared to ND capsules, and liquid chromatography-tandem mass spectrometry (LC-MS/MS) analysis showed 62\% homology with type-1 cytokeratin. Immunohistochemistry using anti-pan cytokeratin antibody revealed co-localization with concanavalin A, and a lectin blot revealed with concanavalin A showed a band of $\sim 65$ $\mathrm{kDa}$, a molecular weight that corresponds to human type 1 cytokeratin.

Conclusion: These results suggest that over-expression of $\mathrm{N}$-glycosidically linked human type 1 cytokeratin may induce capsule disruption and affect selective permeability, allowing the entry of different molecules to the lens that facilitate cataract progression.

Keywords: glycosylation, human epithelial lens cells, diabetes mellitus, cataract

\section{Introduction}

Cataracts are the main cause of reversible blindness globally. ${ }^{1}$ They manifest as a progressive decrease of visual acuity, and the only available treatment is surgical extraction. $^{2}$ The main risk factor for cataract formation is aging, but other factors can induce cataract development, such as trauma, Down syndrome, and myotonic dystrophy, to name a few. After aging, diabetes is one of the most significant risk factors for cataract formation. ${ }^{3}$

Type 2 diabetes mellitus (T2DM) is a chronic systemic disease that can affect all ocular structures, including the lens. Cataract formation in diabetic patients correlates with disease duration. ${ }^{4,5}$ There are several mechanisms responsible for cataract 
formation in T2DM; one of the most studied is overproduction of sorbitol that plays an important role in increasing the osmotic pressure of the lens, causing loss of transparency. ${ }^{6}$ However, there is increasing evidence indicating that lens capsule alterations are also responsible for cataract development.

The lens capsule is a basal membrane (BM) secreted by lens epithelial cells (LECs). The BM and LECs form a continuous barrier that surrounds the entire lens. The primary functions of this barrier are physical protection, promotion of lens differentiation, and regulation of selective permeability to various growth factors, inflammatory mediators, and nutrients, among other molecules. During aging and in certain metabolic disorders like T2DM, chemical and biomechanical properties of the capsule are affected, disrupting selective permeability and allowing the entry of different molecules to the lens. ${ }^{7,8}$ Specifically, the formation of advanced glycation end products (AGEs) potentially leads to lens fiber damage ${ }^{9}$ due to alterations in lens capsule permeability. Glycosylation also seems to be an important modulator of the inflammatory response in various disorders such as diabetes, cancer, and neurodegenerative diseases. ${ }^{10}$ In the eye, glycosylation has been associated with diseases with an inflammatory component, such as dry eye. ${ }^{11}$ Regarding cataracts, recent studies showed an increase of proinflammatory cytokines in the aqueous humor of cataract patients, suggesting that intraocular inflammation plays a role in the pathogenesis of cataracts. ${ }^{12-14}$

Based on the above, since glycosylation is related to several mechanisms that induce cataract formation, ${ }^{15-17}$ we aimed to evaluate glycosylation patterns in human lens capsules from T2DM and ND patients.

\section{Methods}

\section{Design and Setting}

This experimental study used anterior capsules from T2DM and ND patients undergoing routine cataract surgery at the Anterior Segment Department of the Asociación para Evitar la Ceguera en Mexico I.A.P., Hospital "Dr. Luis Sánchez Bulnes", in Mexico City, Mexico. The Institutional Internal Review Board approved this study, which was conducted following the tenets of the Declaration of Helsinki. Informed consent was obtained from all patients before any study procedure.

\section{Samples}

Anterior lens capsules were obtained by continuous circular capsulorhexis during routine phacoemulsification. All patients were free from other ocular diseases and were graded for cataract severity (NO1NC1 to NO5NC5) using the Lens Opacities Classification System III (LOCS III). ${ }^{18}$ We obtained a total of 24 anterior capsules from 24 patients; 12 were used for protein expression (6 from T2DM patients and 6 from ND patients), and 12 for microscopic examination ( 6 from T2DM patients and 6 from ND patients).

\section{Hematoxylin-Eosin Staining}

Lens capsules were immersed in Zamboni fixative for 24 hours at $4^{\circ} \mathrm{C}$. Tissues were mounted on silane-coated slides, then rehydration was performed, and finally they were stained with hematoxylin and eosin using conventional histological techniques and coverslipped using a synthetic mounting media. ${ }^{19}$

\section{Tricine-SDS-PAGE Electrophoresis}

After pulverizing the capsules in a mortar with liquid nitrogen and adding $20 \mu \mathrm{L}$ of buffer with $2 \mu \mathrm{L}$ of a protease inhibitor, samples were centrifuged at 13,000 rpm and $4^{\circ} \mathrm{C}$ for $30 \mathrm{~min}$. After this time, the pellet was discarded and the supernatant was recovered. Then, tricine-sodium dodecyl sulphate-polyacrylamide gel electrophoresis ([tricine-SDS-PAGE) was performed using the method described by Ref. ${ }^{20}$ A constant voltage of $70 \mathrm{~V}$, $100 \mathrm{~mA}$ was applied for $15 \mathrm{~min}$ followed by $120 \mathrm{~V}, 100$ $\mathrm{mA}$ for $75 \mathrm{~min}$. After electrophoresis, the gel and nitrocellulose paper were incubated in a transfer buffer $(25 \mathrm{mM}$ Tris base, $192 \mathrm{mM}$ glycine, and 20\% methanol, $\mathrm{pH} 8.3$ ) for $90 \mathrm{~min}$. Transfer was carried out in a semi-dry system (Bio-Rad, Richmond, CA, USA) at $25 \mathrm{~V}$ for 1 hour.

\section{Immunohistochemistry}

Lens capsules were immersed in Zamboni fixative and mounted on silane-coated slides. The slides were rehydrated using conventional histological techniques. Nonspecific binding sites were blocked by incubation with 2\% IgG-free bovine serum albumin (BSA, SigmaAldrich), followed by permeabilization with $0.2 \%$ Triton X-100 (Sigma). Lectins were diluted at 1:100 with $1 \%$ $\mathrm{BSA}$ in PBS. Incubations were made at $4^{\circ} \mathrm{C}$ overnight. DAPI was used as nuclear counterstain. Biotinylated lectins used were concanavalin A (Con A, Mannose specific) 
ND

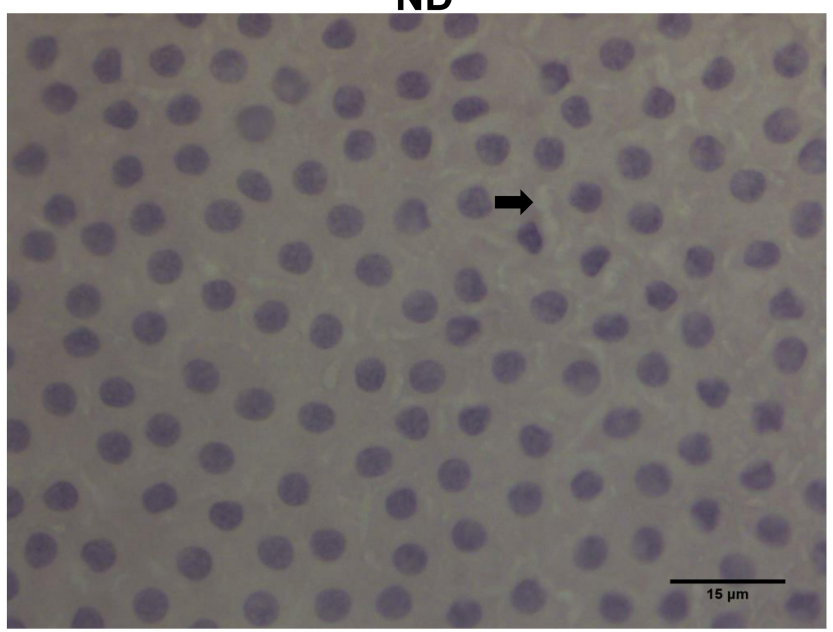

T2DM

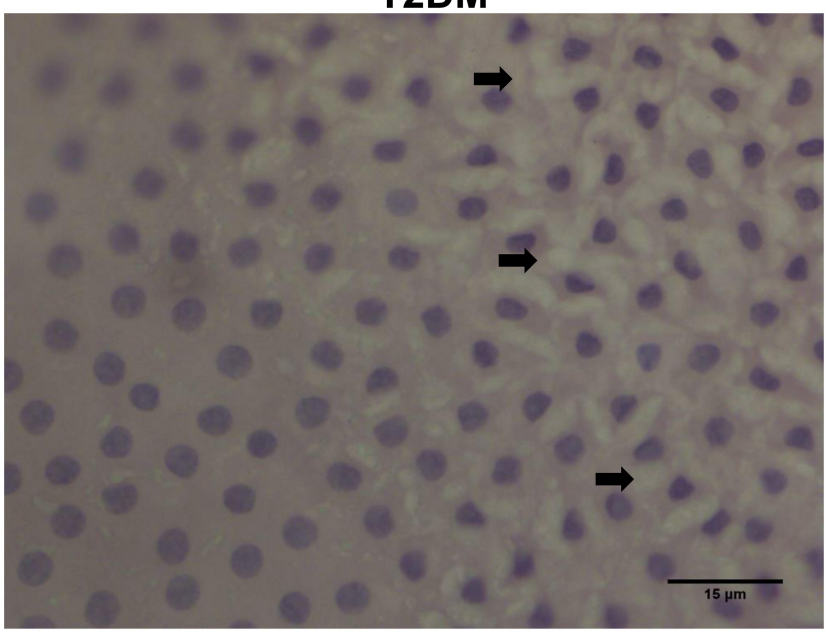

Figure I Hematoxylin-eosin staining (40x) showing cell retraction and cytoplasmic vacuolization (black arrows), sparse in ND and abundant in T2DM samples. Abbreviations: ND, non-diabetic; T2DM, type-2 diabetes mellitus.

(Sigma-Aldrich), jacalin (Galactose/N-acetyl-galactosamine specific, Vector Labs, CA, USA), and PNA (Galactose-specific, peanut agglutinin, Vector Labs). Anti-pan cytokeratin antibody (Abcam, Cambridge UK) was diluted 1:200. Immunostained cells were counted with a Leica DM/LS fluorescent microscope at 40x (Leica Microsystems, Wetzlar, GmBH, Germany).

\section{Two-Dimensional Electrophoresis}

For first dimension analysis, $50 \mu \mathrm{g}$ of protein from the capsule lysates were treated with a mixture of $8 \mathrm{M}$ urea, 0.5\% CHAPS, $0.2 \%$ dithiothreitol, IPG buffer $\mathrm{pH} 3-10$ and $0.002 \%$ bromophenol blue, for a final volume of $130 \mu \mathrm{L}$. Samples were loaded into the immobilized $\mathrm{pH}$ gradient strips, for room temperature incubation during $12 \mathrm{~h}$. Subsequently, the strips were placed in the Amersham Biosciences Multiphor II chamber. An isoelectric focusing was carried out in three phases: the first one at $200 \mathrm{~V}$ for 1 $\mathrm{min}$, at 0.001 kilovolts per hour $(\mathrm{kVh})$ and the second at 3500 $\mathrm{V}$ for $90 \mathrm{~min}$, at $2.8 \mathrm{kVh}$, and finally at $3500 \mathrm{~V}$ for $35 \mathrm{~min}$ at $2.2 \mathrm{kVh}$. For second dimension separation, the strips were soaked in $10 \mathrm{~mL}$ of an equilibration buffer $(50 \mathrm{mM}$ Tris- $\mathrm{HCl}$ $\mathrm{pH}$ 8.8, $6 \mathrm{M}$ urea, 30\% glycerol, 2\% SDS, and 0.002\% bromophenol blue and $65 \mathrm{mM}$ DTT) for $15 \mathrm{~min}$ at room temperature, followed by incubation during 15 min with 10 $\mathrm{mL}$ of a buffer with $135 \mathrm{mM}$ iodoacetamide. The strips were then placed in $10 \%$ SDS-polyacrylamide gels and run at 200 $\mathrm{V}$ for $40 \mathrm{~min}$. Gels were fixed in a solution of $10 \%$ methanol and $7 \%$ acetic acid. Photographs were taken with an Olympus E-500 camera (Japan) and processed with ImageJ.

\section{Liquid Chromatography with Tandem Mass Spectrometry (LC-MS/MS)}

After visualization, target protein spots were excised from the Coomassie stained 2D-SDS PAGE, and they were distained, washed, digested with modified porcine trypsin (Promega, Madison, WI), and extracted as previously described. $^{21}$ The volume of the extracts was reduced by evaporation in a vacuum centrifuge at room temperature and then adjusted to $20 \mu \mathrm{L}$ with $1 \%$ formic acid. Mass spectrometric analysis was carried out in a 3200 QTRAP hybrid tandem mass spectrometer (Applied Biosystems/ MDS Sciex, Concord, ON, Canada), equipped with a nanoelectrospray ion source (NanoSpray II) and a MicroIonSpray II head. The instrument was coupled on line to a nanoAcquity Ultra Performance LC (Waters Corporations, Milford, MA, USA). Samples were desalted by injection onto a Symmetry C18 UPLC trapping column $(5 \mu \mathrm{m}, 180 \mu \mathrm{m}$ x $20 \mathrm{~mm}$, Waters Corporations) and washed with $0.1 \%$ formic acid in Milli Q water. Spectra were acquired in automated mode using Information Dependent Acquisition (IDA). The scan range for EMS was set at m/z 300-1500 and $4000 \mathrm{amu} / \mathrm{s}$, with an ion spray voltage of $+2.2 \mathrm{kV}$ applied to a Picotip emitter FS150-20-10-N (New Objective, Woburn, MA). Data interpretation and protein identification were performed from the MS/MS spectra datasets using the MASCOT search algorithm (Version 1.6b9, Matrix Science, London, UK, available at http://www.matrixscience.com). Searches were conducted using the National Center for Biotechnology Information non-redundant database (NCBIprot, http://www.ncbi. nih.gov). 

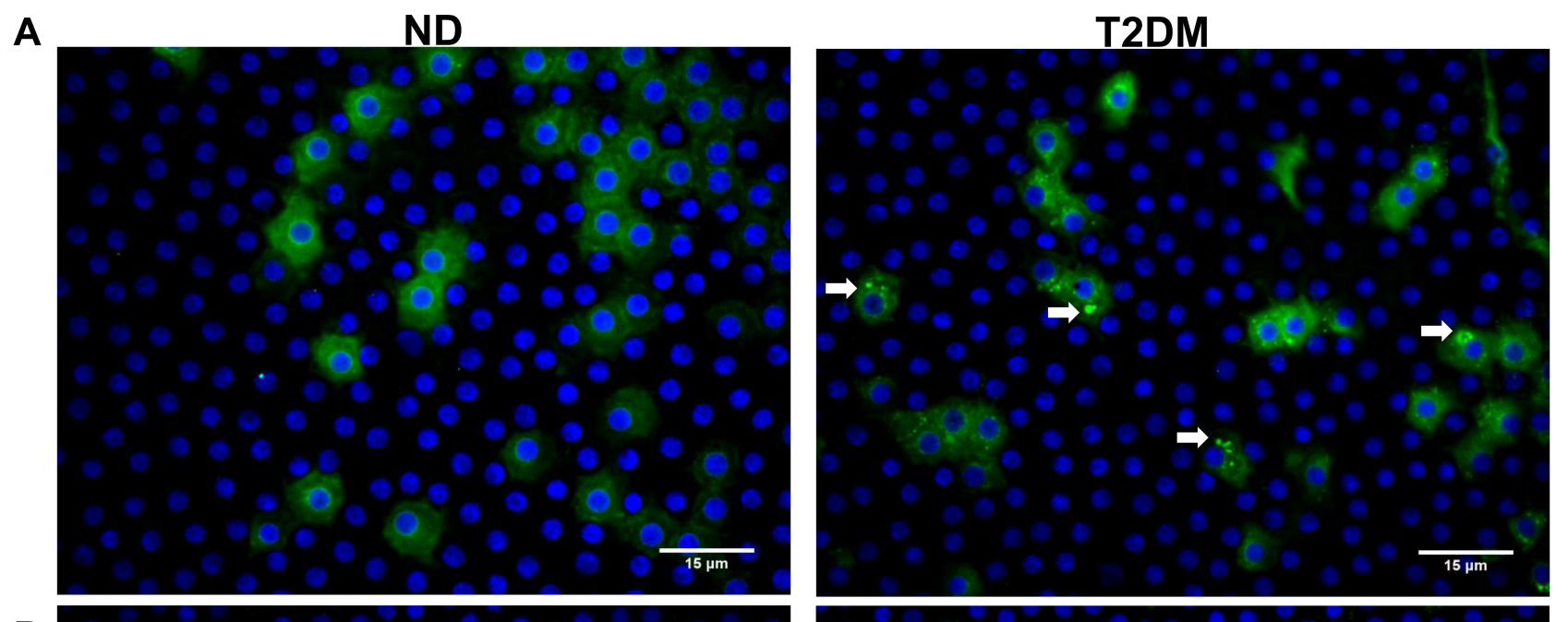

B
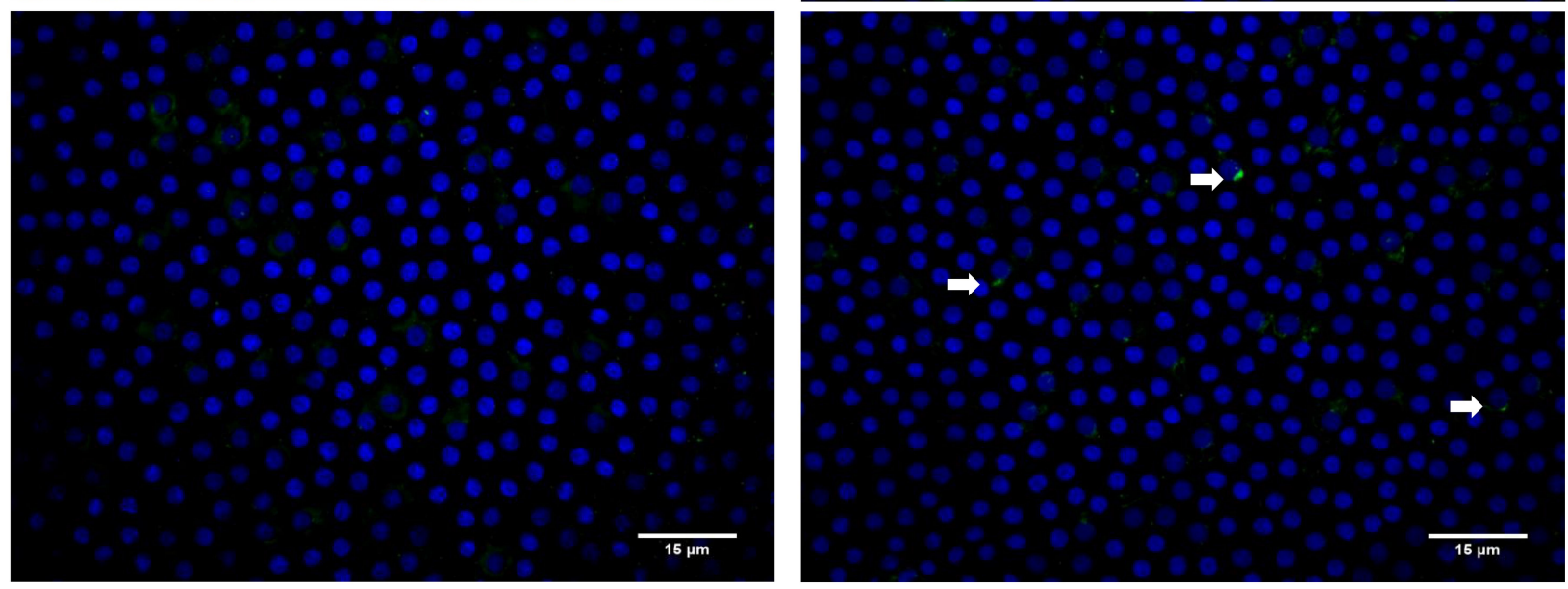

Figure 2 Immunohistochemistry (40x) in ND and T2DM samples using Con A-FITC (green) and DAPI as nuclear counterstain. (A) Con A membrane and cytoplasmic labeling is evident in both ND and T2DM samples; T2DM samples additionally show perinuclear vesicles (white arrows) that, (B) after trypsin treatment during 6 hours at $37^{\circ} \mathrm{C}$, show a certain degree of resistance to digestion.

Abbreviations: ND, non-diabetic; T2DM, type-2 diabetes mellitus.

\section{Results}

Hematoxylin and eosin staining of the anterior capsule showed LECs alterations in T2DM samples, namely loss of intercellular junctions, evidenced by cell retraction and epithelial discontinuity, as well as cytoplasmic vacuolization (Figure 1). To explore the relationship of glycosylated proteins with these histologic changes, we performed immunohistochemistry with the lectin Con A, observing differences in staining intensity between groups. For ND, Con A staining was homogenous both in the cytoplasm and in the membrane but for T2DM, in addition to membrane and cytoplasmic staining, we observed perinuclear vesicles with high reactivity to Con A (Figure $2 \mathrm{~A}$ ). We explored other lectins like jacalin (specific only for O-glycosidically linked oligosaccharides containing galactose/N-acetyl-galactosamine) and PNA (specific for terminal galactose residues), observing no differences between groups (data not shown). When capsules were treated with trypsin for more than 6 hours, Con A labeling decreased considerably; however, ConA positive vesicles in T2DM samples showed a slight decrease, suggesting resistance to trypsin treatment (Figure 2B).

These observations led us to examine the electrophoretic profile between both groups. As depicted in Figure 3, we observed differences between protein extracts from T2DM and ND lysates with Coomassie blue staining, observing a density increase of the 147, 64, and $23 \mathrm{KDa}$ bands in T2DM capsules. After these findings, we performed a 2D-PAGE that evidenced protein spots from 250 to $15 \mathrm{kDa}$ with areas of higher concentration in the isoelectric points from 4 to 8 . In T2DM samples, we observed a spot with a higher density compared to ND samples of $\sim 70 \mathrm{KDa}$, with an isoelectric point of 5.5 (Figure 4A). LCMS/MS analysis using the MASCOT algorithm and the 
A

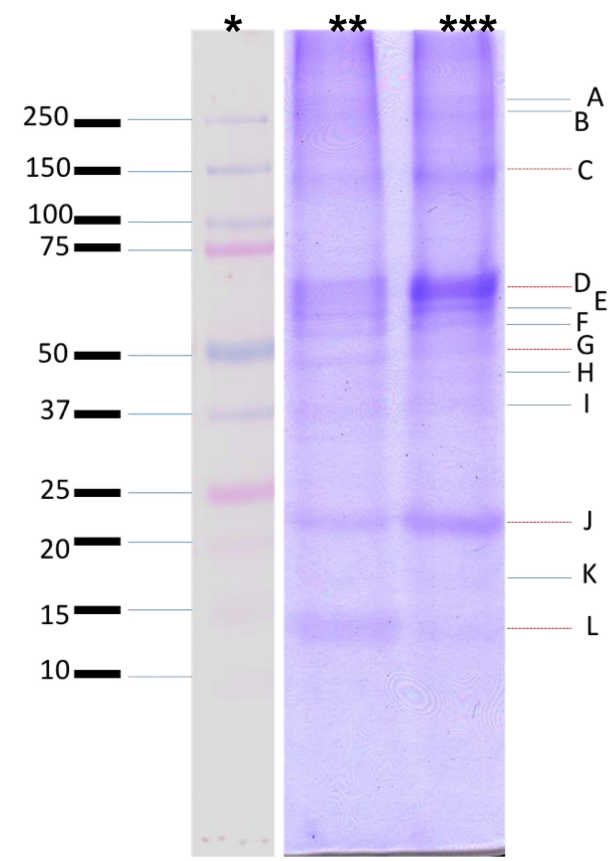

B

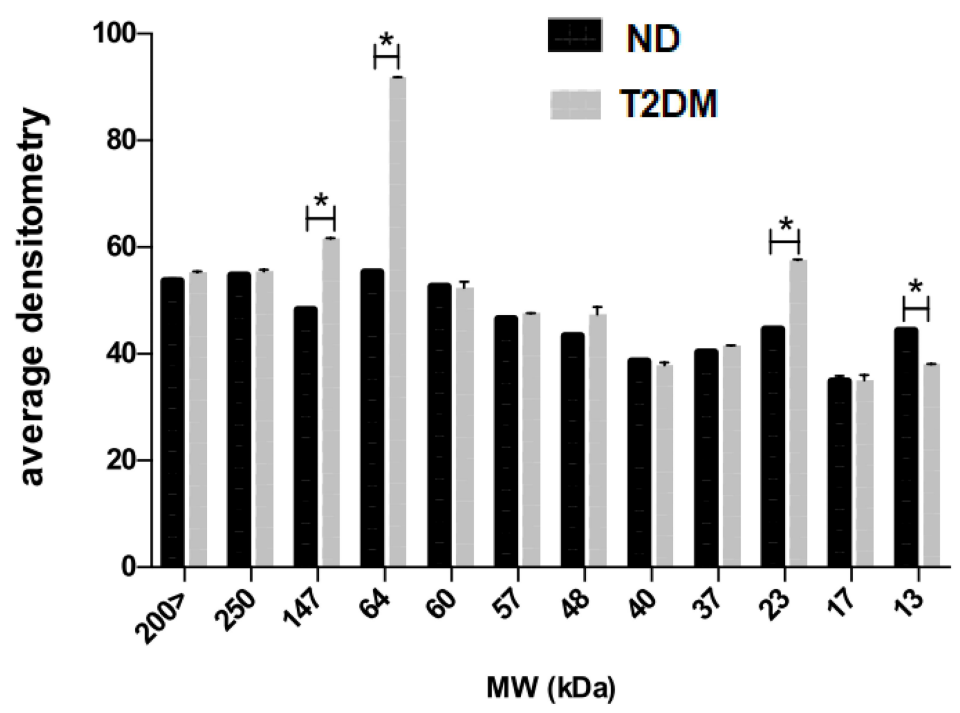

Figure 3 (A) Tricine-SDS-PAGE gel stained with Coomassie blue showing molecular weight (MW) markers (*), a lysate from ND capsules (**) and a lysate from T2DM capsules (***). There is an increased band density for 147, 64 and $23 \mathrm{kDa}$ bands in the T2DM lysate. (B) Relative densitometry quantification shows increased density (with statistical significance) in T2DM samples for the 13, 23, 64 and $147 \mathrm{kDa}$ bands. Y: average density in arbitrary densitometry units, X: molecular weight in kDa. Data expressed as mean $\pm \mathrm{SD}$; unpaired $t$-test with Welch's correction $\left({ }^{*} \mathrm{p}<0.05\right)$.

Abbreviations: ND, non-diabetic; T2DM, type-2 diabetes mellitus.

National Center for Biotechnology Information non-redundant (NCBInr) database of the proteolytic fragments showed a $62 \%$ homology with human cytokeratin (CK) types 1 and 2 (Figure 4B).

To further explore if CK-1/2 had a relationship with the previously described glycosylation-induced alterations, we performed double labeling with ConA-FITC and an anti-pan-cytokeratin antibody conjugated to rhodamine in samples from both groups. We observed colocalization of ConA with anti-pan-CK in both samples; however, T2DM samples showed a stronger co-localization, specifically with the same vesicular pattern observed by immunohistochemistry (Figure 5A). Finally, we performed a lectin blot with biotinylated Con A using capsule homogenates from both groups that confirmed the presence of a $\sim 65 \mathrm{kDa}$ band in T2DM samples, not detected in ND samples (Figure 5B), suggesting the presence of glycosylated CK-1/2 in T2DM lysates. To confirm whether CK- $1 / 2$ has glycosylation sites, we conducted a NetNGlyc 1.0 server analysis to identify potential N-glycosylation sites, indicating that type-1 cytokeratin may be glycosylated at the Asp 410 site (Figure 5C).

\section{Discussion}

Senile and metabolic cataracts show different demographics and progression patterns. T2DM patients show a relatively faster progression and are diagnosed at an earlier age compared to ND patients with senile cataracts. ${ }^{22,23}$ These differences are believed to be secondary, among other factors, to lens capsule alterations that facilitate cataract development. ${ }^{7,24-28}$ It has been shown that alterations in capsule proteins induce loss of transparency of lens fibers and cataract formation, as in the case of patients with Marfan syndrome who have a mutation the glycoprotein fibrillin- $1,{ }^{29}$ as well as mutations in laminin- $\beta 2^{30}$ and collagen IVa5, that result on fragile and thinner capsules and cataract formation. ${ }^{31}$ Another analysis revealed that mice deficient for the glycoprotein SPARC also develops cataracts; these mice show an abnormal capsule morphology with protrusions and increased capsule permeability to macromolecules. ${ }^{32,33}$ As demonstrated in this work, capsules from T2DM patients show changes like lens epithelial cell disruption and cytoplasmic vacuolization indicative of intercellular junctions loss and epithelial discontinuity that suggest adaptive responses for damage limitation. $^{34}$ 

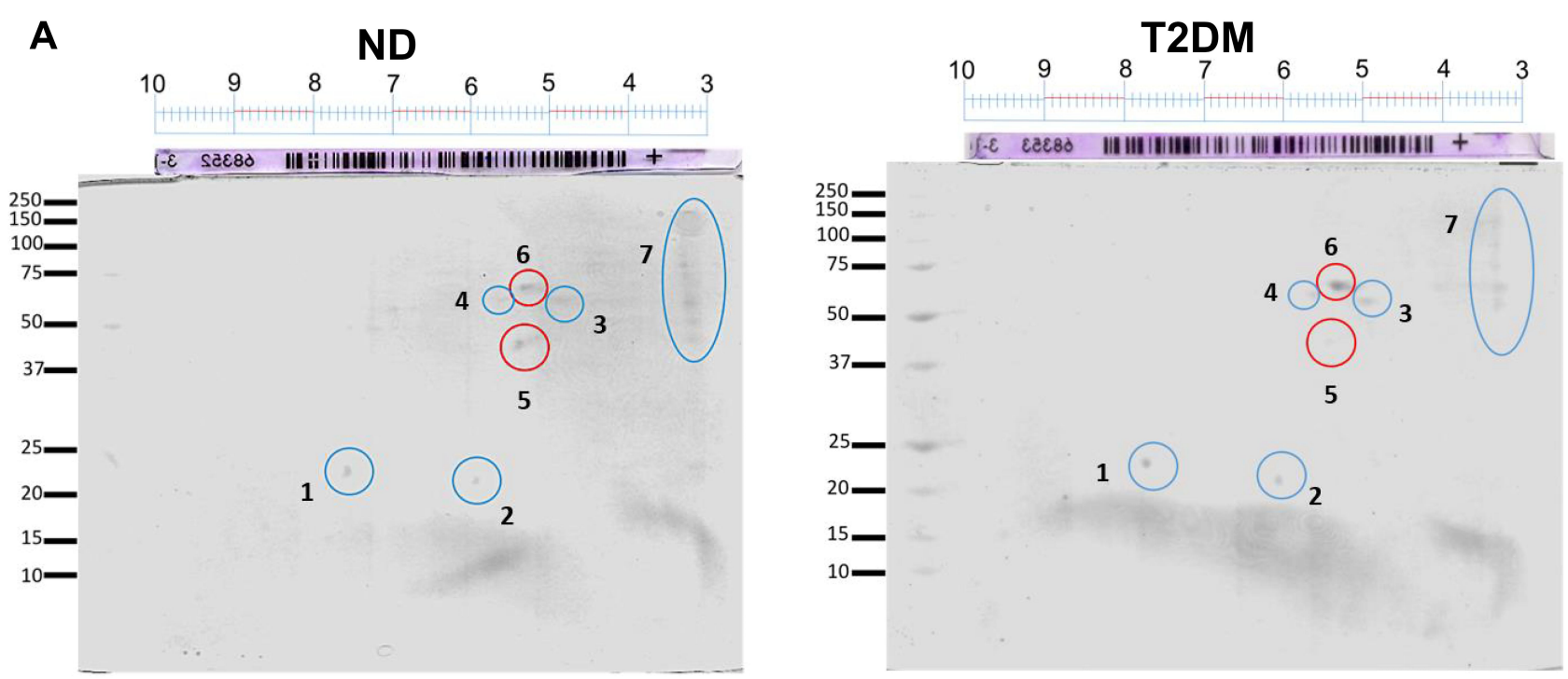

B

\begin{tabular}{|c|c|c|c|c|c|c|c|c|c|c|c|}
\hline 1 & Accession & Description & Score & Coverage & Proteirn & nique Pepti & Peptide & \# PSMs & \# AAs & MW [kDa] & calc. pI \\
\hline 2 & P04264 & Keratin, type II cytoskeletal 1 OS=Homo sapiens i & 531.71 & 62.11 & 1 & 41 & 49 & 240 & 644 & 66.0 & 8.12 \\
\hline 3 & P35527 & Keratin, type I cytoskeletal 9 OS=Homo sapiens G & 484.22 & 62.92 & 1 & 33 & 34 & 191 & 623 & 62.0 & 5.24 \\
\hline 4 & P35908 & Keratin, type II cytoskeletal 2 epidermal OS=Hom & 343.17 & 58.53 & 1 & 35 & 46 & 170 & 639 & 65.4 & 8.00 \\
\hline 5 & P13645 & Keratin, type I cytoskeletal 10 OS=Homo sapiens & 309.25 & 53.42 & 1 & 29 & 36 & 140 & 584 & 58.8 & 5.21 \\
\hline 6 & P04259 & Keratin, type II cytoskeletal 6B OS=Homo sapiens & 258.51 & 53.90 & 1 & 3 & 38 & 133 & 564 & 60.0 & 8.00 \\
\hline 7 & P02533 & Keratin, type I cytoskeletal 14 OS=Homo sapiens & 248.54 & 63.35 & 1 & 13 & 39 & 111 & 472 & 51.5 & 5.16 \\
\hline 8 & P08779 & Keratin, type I cytoskeletal 16 OS=Homo sapiens & 222.21 & 58.56 & 1 & 16 & 33 & 113 & 473 & 51.2 & 5.05 \\
\hline 9 & P02538 & Keratin, type II cytoskeletal 6A OS=Homo sapiens & 212.57 & 55.67 & 1 & 3 & 40 & 110 & 564 & 60.0 & 8.00 \\
\hline 10 & QU4693 & Keratın, type 1 cytoskeletal 1/ US=Homo sapiens & 162.36 & 49.31 & 1 & 11 & 31 & 80 & 432 & 48.1 & 3.02 \\
\hline
\end{tabular}

Figure 4 Two-dimensional electrophoresis of ND capsules and T2DM capsule lysates (A) showing a differential spot of approximately 70 KDa with an isoelectric point of 5.5 (spot number 6). (B) LC-MS/MS analysis using the MASCOT algorithm and the NCBInr database showing a $62 \%$ homology with human cytokeratin types I and 2. Abbreviations: ND, non-diabetic; T2DM, type-2 diabetes mellitus.

In the eye, glycosylation has also been associated with dry eye disease due to a deficiency in MUC5AC mucin glycosylation. ${ }^{11}$ Also, in pterygium, there was an abnormal sialylation of the MUC1 glycoprotein that contributes to neoplastic growth. ${ }^{35,36}$ Therefore, we considered it interesting exploring the relationship between lens epithelial cells alterations and glycosylation using lectins since they are widely studied proteins that recognize specific carbohydrates and glycoproteins; jacalin and PNA are specific for O-glycosylation and B-galactose-containing structures, while Con A shows high specificity for $\alpha$-mannose and $\alpha$ mannose-containing structures such as $\mathrm{N}$-glycosidically linked proteins. ${ }^{37,38}$ As previously described, Con A immunohistochemistry suggested that where there seems to be LEC damage in T2DM patients, there is a potential synthesis of de novo N-glycosylated proteins, in contrast to ND patients. This is consistent with other studies that have observed a relationship between $\mathrm{N}$-glycosylation alterations and diabetes, where metabolic stress induces deregulation of glycan transferases. ${ }^{39}$
From the relevant $\mathrm{N}$-glycosylated proteins detected, we chose to characterize the $\sim 65-\mathrm{kDa}$ protein since it showed the greatest statistically significant difference between groups. 2D-PAGE confirmed the presence of this protein in T2DM samples, and LC-MS/MS confirmed a $62 \%$ homology with CK types 1 and 2. When exploring the role of CK-1/2 with the presumptive glycosylationinduced alterations, we found co-localization of ConA with the anti-pan cytokeratin antibody, and further testing through a lectin blot revealed that Con A recognizes a band of $\sim 65 \mathrm{kDa}$ that potentially corresponds to CK-1/2. Finally, we confirmed by the NetNGlyc 1.0 server that CK-1 has a potential glycosylation site.

The cytoskeleton is a key factor for the proper functioning of the lens, maintaining both cell organization and optical properties. ${ }^{40}$ Indeed, cataracts show cytoskeleton alterations, especially related to intermediate filaments, causing loss of cell morphology that contributes to cataract progression. ${ }^{41}$ Therefore, certain post-translational modifications of cytoskeletal proteins could affect their correct 
A

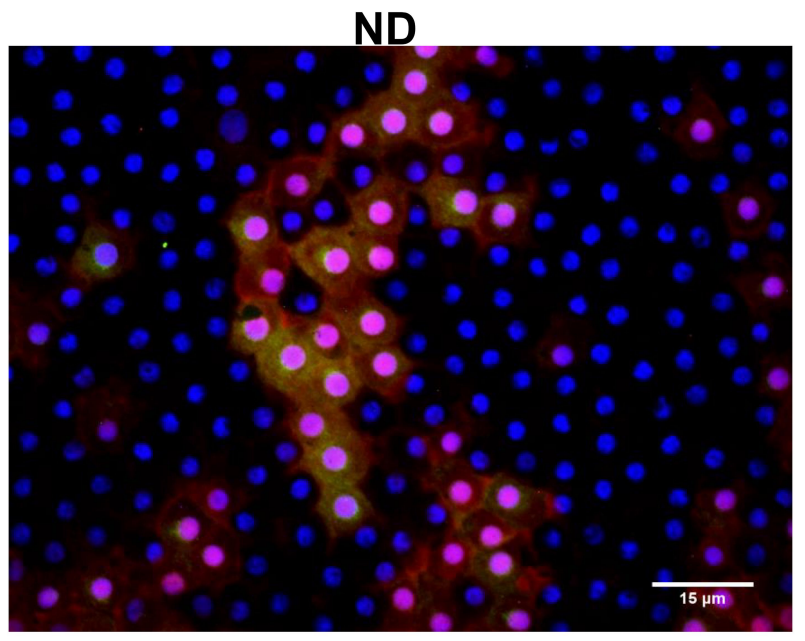

T2DM

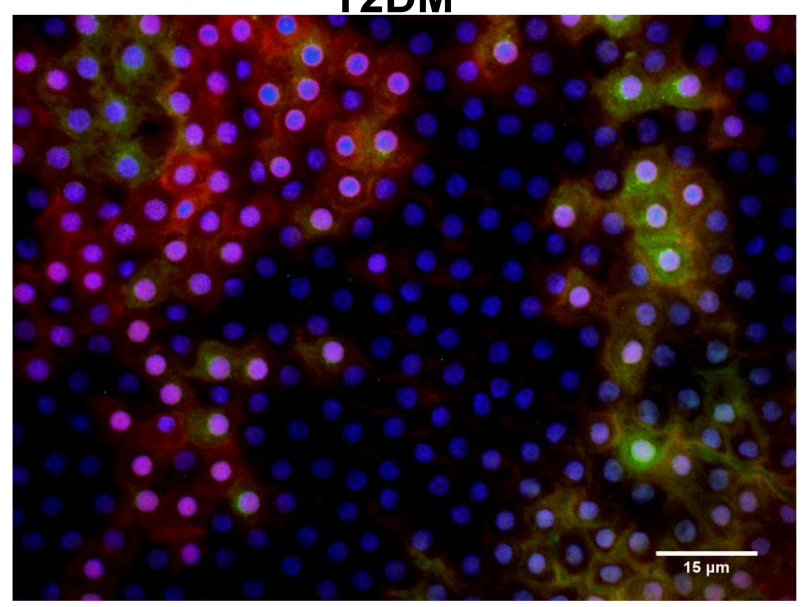

B

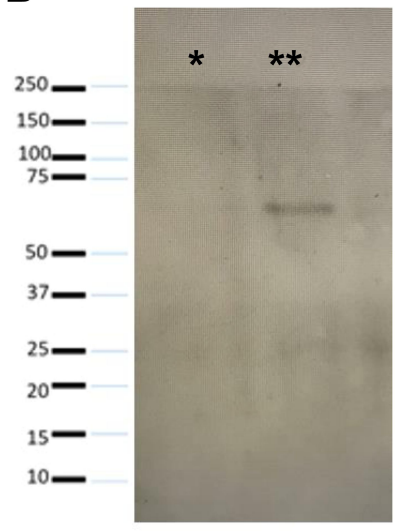

C

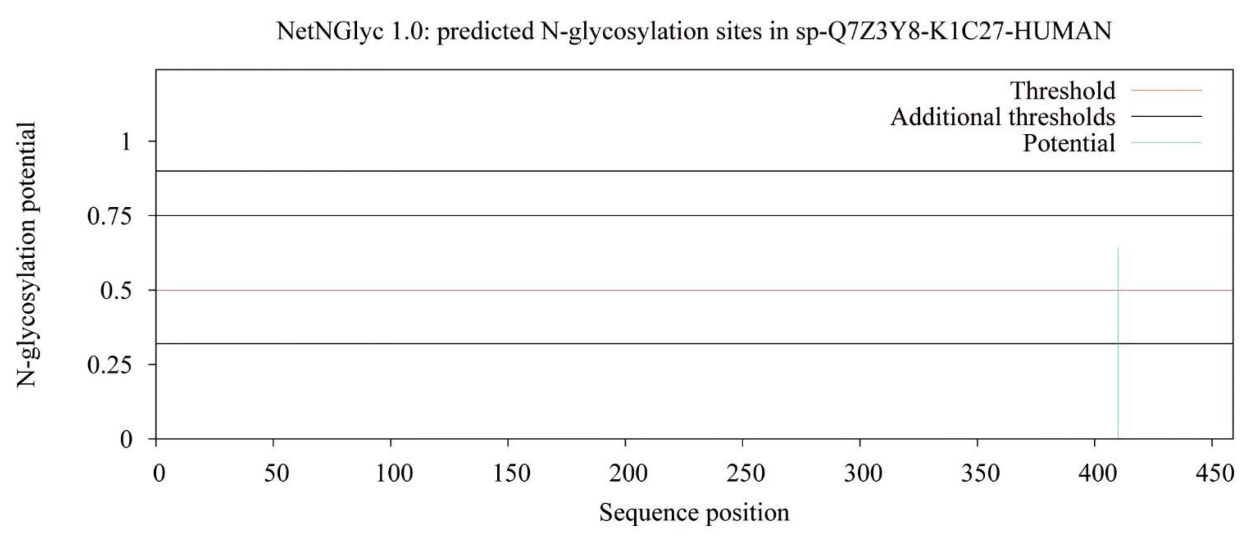

Figure 5 (A) Con A-FITC (green) and anti-pan-cytokeratin (red) co-localization, with DAPI as nuclear counterstain, confirming co-localization both in ND and T2DM samples, more evident in the latter. Observe co-localization at the cytoplasmic vesicular level. (B) Lectin blot revealed with biotinylated ConA in ND (*) and T2DM (**) samples, observing a $60-55 \mathrm{kDa}$ band in the latter. (C) Type I cytokeratin sequence analysis with the NetNGlyc I.0 server. The graph depicts potential N-glycosylation sites throughout the polypeptide chain ( $x$-axis represents the length of the $\mathrm{N}$ - to $\mathrm{C}$-terminal protein; $\mathrm{y}$-axis represents thresholds greater than 0.5 that indicate a high probability of glycosylation). Magnification 40x.

Abbreviations: ND, non-diabetic; T2DM, type-2 diabetes mellitus.

functioning; specifically, glycosylation affects cytoskeleton structure, necessary to maintain lens transparency. An interesting finding of this study was the vesicular localization of Con A observed by IHQ, in contrast to the homogeneous membrane localization observed in ND patients. It has been reported that glycosyltransferases can interact with cytoskeletal proteins, such as the case of core $1 \beta 3$ galactosyltransferase (C1GalT1) that interacts with giantin, and core $2 \mathrm{~N}$-acetyl-glucosaminyltransferase (C2GnT$\mathrm{M})$ that interacts with CK- $1,{ }^{42}$ forming complexes on RER vesicles and the Golgi apparatus, which are important for protein transit and recycling. It is unknown if such glycosyltransferase-cytoskeletal interactions exist or are altered under pathological conditions, but our results suggest that in T2DM, N-glycosylation of cytoskeletal proteins is potentially affecting intermediate filaments, and thus contributing to cataract progression.

Currently, many studies are focused on glycosylation as a therapeutic target, using glycosylated markers for diagnostics, and glycan characterization for drug screening. Also, glycosylation seems to be an important modulator of the inflammatory response in various disorders such as diabetes, cancer, or neurodegenerative diseases. ${ }^{10,43}$ At present, it is unknown how diabetes alters glycosylation, but it has been hypothesized that it could be a direct result of metabolic stress and/or alteration of the hexosamine pathway or secondary to the inflammatory process that affects the expression of glycosyltransferases.

The main limitation of our study is the sparse number of samples, due to the fact that most patients in our tertiary 
care setting have ocular comorbidities, and we decided to exclude patients with ocular disorders different from cataracts in both groups. Nevertheless, we believe that our results strongly suggest that cytoskeleton-associated glycosylation is a relevant mechanism for cataract development in T2DM patients, but further studies are required to identify the specific role of $\mathrm{N}$-glycosylation in cataract occurrence and progression.

\section{Acknowledgments}

Special thanks to Dr. Roberto González Salinas for fruitful discussions and donation of Anterior Lens Capsules to carry out this work. Ivan Emmanuel Ramos Martínez thanks CONACYT for the postdoctoral fellowship (442504).

\section{Funding}

This work was supported in part by the PAPIIT Program from Universidad Nacional Autónoma de Mexico (IN213818) and of the Asociación para Evitar la Ceguera en Mexico I.A.P., Hospital "Dr. Luis Sánchez Bulnes”, in Mexico City, Mexico.

\section{Disclosure}

Rosario Gulias-Cañizo is currently an employee of Alcon Laboratories outside of this work. The authors report no other conflicts of interest in this work.

\section{References}

1. Starr C, Gupta P, Farid M, et al. An algorithm for the preoperative diagnosis and treatment of ocular surface disorders. $J$ Cataract Refract Surg. 2019;45(5):669-684. doi:10.1016/j.jcrs.2019.03.023

2. Sheeladevi S, Lawrenson J, Fielder A, Suttle C. Global prevalence of childhood cataract: a systematic review. Eye. 2016;30:1160-1169.

3. Thompson J, Lakhani N. Cataracts. Prim Care Clin Office Pract. 2015;42:409-423. doi:10.1016/j.pop.2015.05.012

4. Kim SI, Kim SJ. Prevalence and risk factors for cataracts in persons with type 2 diabetes mellitus. Korean J Ophthalmol. 2006;20(4):201204. doi:10.3341/kjo.2006.20.4.201

5. Pollreisz A, Schmidt-Erfurth U. Diabetic cataract—pathogenesis, epidemiology and treatment. J Ophthalmol. 2010;2010.

6. Bessa A, Ragab A, Nassra R, Piñero D, Shaheen S. Expression levels of aldose reductase enzyme, vascular endothelial growth factor, and intercellular adhesion molecule-1 in the anterior lens capsule of diabetic cataract patients. J Cataract Refract Surg. 2018;44(12):14311435. doi:10.1016/j.jcrs.2018.07.054

7. Fisher RF. Changes in the permeability of the lens capsule in senile cataract. Trans Ophthalmol Soc UK. 1977;97:100-103.

8. Krag S, Andreassen T. Mechanical properties of the human lens capsule. Prog Ret Eye Res. 2003;22:749-767.

9. Kiziltoprak H, Tekin K, Inanc M, Sakir Y. Cataract in diabetes mellitus. World J Diabetes. 2019;10(3):140-153. doi:10.4239/wjd.v10. i 3.140
10. Dewald J, Colomb F, Bobowski-Gerard M, Groux-Degroote S, Delannoy P. Role of cytokine-induced glycosylation changes in regulating cell interactions and cell signaling in inflammatory diseases and cancer. Cells. 2016;5:43. doi:10.3390/cells5040043

11. Stephens DN, McNamara N. Altered mucin and glycoprotein expression in dry eye disease. Optom Vis Sci. 2015;92:931-938. doi:10.1097/OPX.0000000000000664

12. Takai Y, Tanito M, Ohira A. Multiplex cytokine analysis of aqueous humor in eyes with primary open-angle glaucoma, exfoliation glaucoma, and cataract. Invest Ophthalmol Vis Sci. 2012;53(1):241-247. doi:10.1167/iovs.11-8434

13. Ten Berge J, Fazil Z, van den Born I, et al. Intraocular cytokine profile and autoimmune reactions in retinitis pigmentosa, age-related macular degeneration, glaucoma and cataract. Acta Ophthalmol. 2019;97(2):185-192. doi:10.1111/aos.13899

14. Zheng Y, Rao YQ, Li JK, Huang Y, Zhao P, Li J. Age-related proinflammatory and pro-angiogenic changes in human aqueous humor. Int J Ophthalmol. 2018;181:96-200.

15. Chen W, Lin H, Zhong X, et al. Discrepant expression of cytokines in inflammation- and age-related cataract patients. PLoS One. 2014;9: e109647.

16. Papadimitriou D, Bothou C, Skarmoutsos F, Alexandrides T, Papaevangelou V, Papadimitriou A. The autoimmune hypothesis for acute bilateral cataract in type 1 diabetes. Diabetes Metab. 2016;42:18. doi:10.1016/j.diabet.2016.04.006

17. Selin JZ, Lindblad BE, Rautiainen S, et al. Are increased levels of systemic oxidative stress and inflammation associated with agerelated cataract? Antioxid Redox Signal. 2014;21(5):700-704. doi:10.1089/ars.2014.5853

18. Chylack LT, Wolfe JK, Singer DM, et al. The lens opacities classification system III. Arch Ophthalmol. 1993;111:831-836. doi:10.1001/ archopht.1993.01090060119035

19. Humason GL. Specific staining methods. In: Kennedy D, Park RB, editors. Animal Tissue Techniques. San Francisco CA: W.H. Freeman and Co; 1972:183-185.

20. Schägger H. Tricine-SDS-PAGE. Nat Protoc. 2006;1(1):16-22. doi:10.1038/nprot.2006.4

21. Solórzano C, Mayoral M, de Los Angeles Carlos M, et al. Over expression of glycosylated proteins in cervical cancer recognized by the Machaerocereuseruca agglutinin. Folia Histochemica Et Cytobiologica. 2012;50:398-406. doi:10.5603/FHC.2012.0054

22. Drinkwater JJ, Davis WA, Davis TME. A systematic review of risk factors for cataract in type 2 diabetes. Diabetes Metab Res Rev. 2019;35:35. doi:10.1002/dmrr.3073

23. Srinivasan S, Raman R, Swaminathan G, Ganesan S, Kulothungan V, Sharma ST. Incidence, progression, and risk factors for cataract in type 2 diabetes. Invest Ophthalmol Vis Sci. 2017;58(13):5921-5929. doi:10.1167/iovs.17-22264

24. De Iongh RU, Wederell E, Lovicu FJ, McAvoy JW. Transforming growth factor-beta-induced epithelial-mesenchymal transition in the lens: a model for cataract formation. Cells Tissues Organs. 2005;179:43-55. doi:10.1159/000084508

25. Duncan MK, Kozmik Z, Cveklova K, Piatigorsky J, Cvekl A. Overexpression of PAX6 (5a) in lens fiber cells results in cataract and upregulation of (alpha) 5 (beta) 1 integrin expression. J Cell Sci. 2000;113:3173-3185.

26. Inanc M, Tekin K, Erol YO, et al. The ultrastructural alterations in the lens capsule and epithelium in eyes with traumatic white cataract. Int Ophthalmol. 2019;39(1):47-53. doi:10.1007/s10792-017-0783-0

27. Wilson J, Trivedi M, Biber R, Golub R. Anterior capsule rupture and subsequent cataract formation in Alport syndrome. $J$ AAPOS. 2006;10:182-183. doi:10.1016/j.jaapos.2005.09.008

28. Yan Y, Yu H, Sun L, et al. Laminin $\alpha 4$ overexpression in the anterior lens capsule may contribute to the senescence of human lens epithelial cells in age-related cataract. Aging. 2019;11(9):2699-2723. doi:10.18632/aging. 101943 
29. Ammash NM, Sundt TM, Connolly HM. Marfan syndrome-diagnosis and management. Curr Probl Cardiol. 2008;33:7-39. doi:10.1016/j. cpcardiol.2007.10.001

30. Zenker M, Aigner T, Wendler O, et al. Human laminin $\beta 2$ deficiency causes congenital nephrosis with mesangial sclerosis and distinct eye abnormalities. Hum Mol Genet. 2004;13(21):2625-2632. doi: $10.1093 / \mathrm{hmg} / \mathrm{ddh} 284$

31. Van Agtmael T, Schlotzer-Schrehardt U, McKie L, et al. Dominant mutations of Col4al result in basement membrane defects which lead to anterior segment dysgenesis and glomerulopathy. Hum Mol Genet. 2008;14:3161-3168. doi:10.1093/hmg/ddi348

32. Yan Q, Clark J, Wight T, Sage H. Alterations in the lens capsule contribute to cataractogenesis in SPARC-null mice. J Cell Sci. 2002;115:2747-2756.

33. Yan Q, Perdue N, Blake D, Sage E. Absence of SPARC in murine lens epithelium leads to increased deposition of laminin-1 in lens capsule. Invest Ophthalmol Vis Sci. 2005;46:4652-4660. doi:10.1167/ iovs.05-0460

34. Henics T, Wheatley DN. Cytoplasmic vacuolation, adaptation and cell death: a view on new perspectives and features. Biol Cell. 1999;91(7):485-498. doi:10.1016/S0248-4900(00)88205-2

35. Kase S, Kitaichi N, Furudate N, Yoshida K, Ohno S. Increased expression of mucinous glycoprotein KL-6 in human pterygium. $\mathrm{Br}$ J Ophthalmol. 2006;90:1208-1209. doi:10.1136/bjo.2006.094300

36. Kawano K, Uehara F, Ohba N. Lectin-cytochemical study on epithelial mucus glycoprotein of conjunctiva and pterygium. Exp Eye Res. 1988;47:43-51. doi:10.1016/0014-4835(88)90022-X
37. Debray H, Decout D, Strecker G, Spik G, Montreuil J. Specificity of twelve lectins towards oligosaccharides and glycopeptides related to N-glycosylproteins. Eur J Biochem. 1981;117(1):41-55. doi:10.1111/ j.1432-1033.1981.tb06300.x

38. Sharma V, Srinivas VR, Adhikari P, Vijayan M, Surolia A. Molecular basis of recognition by Gal/GalNAc specific legume lectins: influence of Glu 129 on the specificity of peanut agglutinin (PNA) towards C2substituents of galactose. Glycobiology. 1998;8:1007-1012. doi:10.1093/glycob/8.10.1007

39. Rudman N, Gornik O, Lauc G. Altered N-glycosylation profiles as potential biomarkers and drug targets in diabetes. FEBS Lett. 2019;593:1598-1615. doi:10.1002/1873-3468.13495

40. Song S, Landsbury A, Dahm R, Liu Y, Zhang Q, Quinlan R. Functions of the intermediate filament cytoskeleton in the eye lens. $J$ Clin Invest. 2009;119:1837-1848. doi:10.1172/JCI38277

41. Tagliavini J, Gandolfil S, Marainil G. Cytoskeleton abnormalities in human senile cataract. Curr Res Eye. 1986;5:903-910. doi:10.3109/ 02713688608995170

42. Petrosyan A, Ali MF, Cheng P. Keratin 1 plays a critical role in golgi localization of core $2 \mathrm{~N}$-acetylglucosaminyltransferase $\mathrm{M}$ via interaction with its cytoplasmic tail. J Biol Chem. 2015;290:6256-6269. doi:10.1074/jbc.M114.618702

43. Harding C, Feldman MF. Glycoengineering bioconjugate vaccines, therapeutics, and diagnostics in E. coli. Glycobiology. 2019;29:519-529.
Clinical Ophthalmology

\section{Publish your work in this journal}

Clinical Ophthalmology is an international, peer-reviewed journal covering all subspecialties within ophthalmology. Key topics include: Optometry; Visual science; Pharmacology and drug therapy in eye diseases; Basic Sciences; Primary and Secondary eye care; Patient Safety and Quality of Care Improvements. This journal is indexed on PubMed
Dovepress

Central and CAS, and is the official journal of The Society of Clinical Ophthalmology (SCO). The manuscript management system is completely online and includes a very quick and fair peer-review system, which is all easy to use. Visit http://www.dovepress.com/ testimonials.php to read real quotes from published authors. 